

\title{
Enhancing the Channel Capacity of a Cellular Mobile System using Frequency Diversity
}

\author{
Aguodoh Patrick C . \\ Michael Okpara. University of Agriculture \\ Umudike, Abia State, Nigeria
}

\author{
Chineke Hyacinth A. \\ Michael Okpara. University of Agriculture \\ Umudike, Abia State, Nigeria
}

\begin{abstract}
Communication industries have been encountering a lot of problems in their communication links some of such difficulties or problems are Interference, fading, overhanging, attenuation and congestion. Transmitted signals with different frequencies are affected in different ways in frequency domain. The fact is an advantage in frequency diversity technique. Multiple replicas of information signal are sent over several affected frequency band in this diversity. In a sufficient wide signal bandwidth, multipath components can resolve. This paper presents the technique that can enhance the channel capacity of a cellular mobile system. Error control coding and interleaving using frequency diversity was used to combat time selective or fast fading in communication link. In the result, it is possible to obtain different independently fading signal. The number of resolvable multipath equation is used to approximate the maximum achievable diversity order for multipath diversity.
\end{abstract}

Keyword: Communication, Space Time Block Code, Space Time Trellis Code, channel capacity

\section{INTRODUCTION}

In the field of communication, wireless communication is the fastest growing technology and the growth of the cellular system is exponential over the last decade. Wireless systems have the capacity to cover broad geographical areas, and it excludes the more costly infrastructures to deploy wired links to the individual sites. The main purpose of broadband wireless communication is to provide reliable and high data rate transmission over a larger area. The broadband wireless systems that provide multimedia services such as high-speed internet access, wireless television, online gaming and mobile computing are rapidly growing. Considering the reliability of wireless channel, the diversity technique is used so that the receiver will get the independently faded copies of the transmitted signal, and the receiver will be able to receive at least one of these replicas correctly. Space Time Coding (STC) is an efficient scheme used in modern wireless communications employing multiple input multiple output(MIMO) system. For the realization of the diversity benefits of multiple transmit antennas, Space Time Coding is used (DUMAN,2015).

In wireless communications, antenna system is designed for reducing multipath fading, interference and polarization mismatch. The multiple antennas deployed at the transmitting and receiving side called MIMO systems, which provide high data rates in rich scattering environments are being implemented in recent communication systems. MIMO system also improves the data transmission reliability and all these benefits of MIMO system can be achieved without increasing the transmission power and the required bandwidth. The spatial diversity obtained from transmission and reception antennas can be combined with the channel coding, and the outcome of the combination is called space-time coding, and the system is referred to as coded MIMO system. Coding over MIMO system provides a solution for reliable high-speed wireless communication links. Generally, space time coding can be classified into two types:

Space Time Block Code (STBC) and Space Time Trellis Code (STTC). Both STBC and STTC are used 
to achieve full spatial diversity in a given number of transmitting and receiving antennas but STTC which is based on transmission achieves spatial diversity as well as coding gains. In STBC, data encoding is done using STBC encoder and the encoded data is converted into $\mathrm{n}$ streams. Then $\mathrm{n}$ streams are simultaneously transmitted over $\mathrm{n}$ transmission antennas. At the receiving side of each antenna, the received signal is the superposition of transmitted signal. The Space Time Block Codes are designed in such a way that maximum diversity order for the number of transmit and receive antennas is achieved. STBC can be decoded more efficiently at the receiver by linear processing. In case of two transmit antennas, Altamonte scheme is used to obtain full transmit diversity, and this technique is comparatively simpler to implement. Here, Rayleigh fading channel is considered as it provides good performance for outdoor and long-distance communication (LIU,2014).

Communication industries have been encountering a lot of problems in their communication links some of such difficulties or problems are Interference, fading, overhanging, attenuation and congestion. These unfortunate situations have made the passage of piece of information from one point to another or from source to sink difficult. This will equally reduce their output to out $40 \%$ thereby discouraging customers not to be subscribing to their network. This ugly condition will equally discourage investors from building factories and industries thereby reducing the economic growth of the nation. The profits of such communication industries will equally reduce drastically. On the other hand if enhancing the channel capacity of a cellular mobile system using frequency diversity is incorporated in our communication industries their output may increase to $20 \%$.

\section{Methodology}

To design a model that reduces bit error rate thereby enhancing the channel capacity of a cellular mobile system using frequency diversity. What is the channel capacity for a teleprinter channel with a100Hz, 200Hz 300Hz, $400 \mathrm{~Hz}, 500 \mathrm{~Hz}$ and $600 \mathrm{~Hz}$ bandwidth and a signal-to-noise ratio of 3 DB?

\section{Solution:}

Using Shannon's equation: $\mathrm{C}=\mathrm{B} \log _{2}(1+\mathrm{SNR})$ we have

$$
\mathrm{B}=100 \mathrm{~Hz} \text { and } \mathrm{SNR}=3 \mathrm{~dB}
$$

Therefore, $\mathrm{SNR}=10^{\wedge} 0.3$

$$
\begin{array}{ll}
\text { To calculate } & \mathrm{C} 1=100 \log 2\left(1+10^{\wedge} 0.3\right) \\
\mathrm{C} 1 & =100 \log 2(1+1.995) \\
\mathrm{C} 1 & =100 \log 2(2.995) \\
\mathrm{C} 1 & =10^{\wedge} 1^{*} 0.476 \\
\mathrm{C} 1 & =4.76 \mathrm{Bps}
\end{array}
$$

To calculate the capacity when the frequency is $200 \mathrm{~Hz}$

$$
\begin{aligned}
& \mathrm{C} 2=200 \log 2(2.995) \\
& \mathrm{C} 2=10^{\wedge} 2 * 0.476 \\
& \mathrm{C} 2=47.6 \mathrm{bps}
\end{aligned}
$$

To find the value of the capacity when the frequency is $300 \mathrm{~Hz}$

$$
\begin{aligned}
& \mathrm{B}=300 \mathrm{~Hz} \text { and } \mathrm{SNR}(\text { in } \mathrm{dB})=3, \\
& \text { Therefore, } \mathrm{SNR}=10^{0.3} \\
& \mathrm{C} 3=300 \log _{2}\left(1+10^{0.3}\right) \\
& \mathrm{C} 3=300 \log _{2}(2.995) \\
& \mathrm{C} 3=10^{\wedge} 3 * 0.476 \\
& \mathrm{C} 3=476 \mathrm{bps}
\end{aligned}
$$

To evaluate the channel capacity when the frequency is $400 \mathrm{~Hz}$

$$
\begin{aligned}
& \mathrm{C} 4=400 \log 2(2.995) \\
& \mathrm{C} 4=10^{\wedge} 4^{*} 0.476 \\
& \mathrm{C} 4=4760 \mathrm{bps}
\end{aligned}
$$

To solve for the channel capacity when the frequency is $500 \mathrm{~Hz}$

$$
\begin{aligned}
& \mathrm{C} 5=500 \log 2(2.995) \\
& \mathrm{C} 5=10^{\wedge} 5^{*} 0.476 \\
& \mathrm{C} 5=47600 \mathrm{bps}
\end{aligned}
$$

To calculate the channel capacity when the frequency is $600 \mathrm{~Hz}$

$$
\begin{aligned}
& \mathrm{C} 6=600 \log 2(2.995) \\
& \mathrm{C} 6=10^{\wedge} 6^{*} 0.476 \\
& \mathrm{C} 6=476000 \mathrm{bps}
\end{aligned}
$$

Let's say that 1,000 bits are transmitted, and a bit, 2 bits, 3 bits, 4 bits, 5 bits, 6 bits, 7 bits and 8 bits received are error because of some kind interference between the transmitter and receiver.

BER in this example is calculated by dividing the number of error red bits by the total number of bits transmitted

$$
\mathrm{BER}=\frac{1}{1,000=0.001}
$$

To find BER at 2 bit error rate 
$\frac{2}{1,000,000}=0.000002$

To find BER at 3 bit error rate

$\mathrm{BER}=\quad$ Errored bits received

Total number of bits

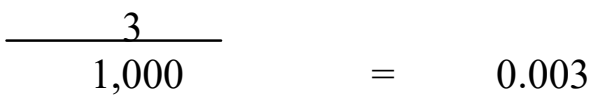

To find BER at 4 bit error rate

$\mathrm{BER}=$ Errored bits received

Total number of bits

$\mathrm{BER}=\frac{4}{1,000=0.004}$

To solve for 5 bit error rate BER

Applying formula for BER $=$ Errored bits received

Total number of bits

$\mathrm{BER}=\frac{5}{1,000}=0.005$

To solve for 6 bit error rate BER

Applying formula for BER $=$ Errored bits received

Total number of bits

$\mathrm{BER}=\frac{6}{1,000=0.006}$

To solve for 7 bit error rate BER

Applying formula for BER $=$ Errored bits received

Total number of bits

$\mathrm{BER}=\frac{7}{1,000}=0.007$

To solve for 8bit error rate BER

Applying formula for BER $=$ Errored bits received

Total number of bits

$$
\mathrm{BER}=\frac{8}{1,000=0.008}
$$

\section{Multipath or Frequency Diversity:}

In a channel, Transmitted signals with different frequencies are affected in different ways in frequency domain. The fact is an advantage in frequency diversity technique. Multiple replicas of information signal are sent over several affected frequency band in this diversity. There should be a distance more than coherent bandwidth between the frequency bands and achieve small-scale fading according to the following equation.
Moreover, frequency hopping (FH) might be used to achieve such kind of diversity instead of sending multiple frequency replicas over different affecting frequencies. Frequency band can be changed many times per symbol in fast frequency hopping (FFH) and this results frequency diversity on each transmitted symbol. This process is very beneficial in an environment where there is a partial band jamming in channel [5]. However, a different frequency band is used for a burst of symbol in slow frequency hopping (SFH). If SFH is combined with time domain coding and interleaving, it is seen as like an additional block interleaving in the frequency domain. This kind of spreading of information in a frequency domain introduces frequency diversity benefit.

Frequency diversity can also be implied as in the case of multipath diversity. Transmission of a wideband signal is given by the following equation where the bandwidth is more than the coherence bandwidth of the previously used channel and this results a frequency selective fading [3].

In a sufficient wide signal bandwidth, multipath components can resolve. In the result, it is possible to obtain different independently fading signal. The number of resolvable multipath given by the following equation is used to approximate the maximum achievable diversity order for multipath diversity.

Channel equalization is another approach to achieve multipath diversity. A filter is used at receiver to make channel equalization to compensate the channel impairments. This process combines the multipath of signals and reduces inter-symbol-interference (ISI) and produce diversity.

In general, the information signals are modulated through different carriers $M$ in frequency diversity scheme. It is important that different signals undergo independent fading. The carriers should be separated by at least coherent bandwidth from each other. L copies of signals are optimally combined at the receiver to make a statistic decision. The maximum ratio combiner is the optimal combiner.

\section{Spatial/Space Diversity:}

Multiple antennas are used to transmit signals carrying information at the transmitter and/or receiver to provide multiple independent fading paths in space 
diversity. This technique is used to provide significant performance gain with not sacrificing any valuable bandwidth on the transmitted power resources. Spatial diversity is widely used because it is easy to implement and it's cost effective an transmitting but multiple receiving antennas. The receiving antennas should be at enough distance for that the multiple fading in the diversity will be uncorrelated. There should be a balanced average power between channels and the correlation coefficient should be very low to achieve a good diversity gain [9]. While wide distance is required between antennas for obtaining low correlation between channels but close distance is also required to synthesize to make a narrow beam not generating grating lobes which prevent introducing interference. Only one or two co-channel interfering signals are used in time division multiple access (TDMA) systems. However, the number of signals is more in code division multiple access (CDMA) the maximal ratio combining (MRC) is better than the performance of optimum adapting processing. It is suggested that if we increase antenna element separation as much as feasible the then high space diversity gain can be achieved with maximal ratio combining in a CDMA system. Most of the cellular communication system has only one transmitting antenna at the base station and two receiving antennas those are widely separated per sector in a space diversity system.

In general, if we want to receive $M$ copies of transmitted signals then we need $M$ number of antennas in a space diversity system (Sarkar,2014). It is very important to keep enough space between the antennas so that the received signals undergo independent fading. Space diversity is different than frequency and temporal diversity. Unlike those spaces diversity needs no additional work at the transmission end and no additional bandwidth is required on the transmission time.

On the other hand, physical complexity restricts its application widely. Like several receiving antennas use in space diversity, several transmission antennas also can be used to send several copies of transmitted signals. This kind of diversity can be employed combating frequency and time selective fading both. There are two types of spatial diversity techniques such as receive diversity and transmit diversity.

\section{Receive Diversity:}

Multiple antennas are use at the receiver to obtain diversity and employ switching and combining or selection intending to improve the quality of received signal. Since it is easier and cost effective to use multiple antennas at the base station than the terminal which is a positive manner of receive diversity. This technique may utilize channel state information (CSI) at receiver and it's fully fit for uplink receive diversity are cost, size and necessary power at the remote units. This technique is larger in size and expensive in cost because of multiple antennas, radio frequency chains or selections and its switching circuits.

\section{Transmit Diversity:}

Unlike receive diversity, transmit diversity needs multiple transmitting antennas. Moreover, unlike receive diversity, transmit diversity does not utilize CSI in its single information signal. Effective signal processing technique should be used to extract the noisy and distorted received signal in transmit diversity.

\section{Time / Temporal Diversity:}

Interleaving and coding, over symbols across different coherent time periods, is used to obtain time or temporal diversity. This technique utilizes coding of channel and interleaving to mitigate channel fading at a cost of added delay and loss of bandwidth efficiency. It is uses on slow fading channels and on the channels which is delay sensitive.

Intentional redundancy is introduced into the transmitted signal to achieve time diversity in the temporal domain. Redundancy can be done by repetition of channel coding. To make repetition coding, information bearing signals are transmitted in several time slots. But the separation between time slots should be more or equal than the coherent time of the channel to obtain independent faded signals which helps to gain full diversity advantages. Moreover, it is possible to obtain repetition coding by spreading in direct-sequence code division multiple access (DS-CDMA).

Channel coding, when there is an error control capability, may be performed separately or with modulation. If we want to perform coding and modulation separately, then we can use conventional error control coding to achieve redundancy in the form of extra symbols. The advantage to this case is that the transmission of extra symbols leads to lower efficiency in system bandwidth. On the other hand, if 
we want to perform coding and modulation together (the process also called as coded modulation) then the redundancy comes from the channel-set expansion. But the most beneficial process is ceded modulation because it is bandwidth efficient since it allows error control coding without increasing the bandwidth.

If the successive channel symbols are independent and the channel is Rayleigh fading then the effective minimum time diversity of the channel code is equal to its lowest hamming distance in signal symbols. But the problem is that if the fading is not very fast then there are no successive and independent channel symbols. As a result we can't achieve the system. Longer fade duration with slower fading results burst errors if there is no use of interleaving or effective burst error corresponding codes. The purpose of the interleaving is to impose further spread out in time coded signal symbols to achieve independent errors at the place of burst error. Therefore, a code is used which is capable to correct errors.

In general, a desired signal is transmitted in $\mathrm{M}$ different periods of time in time diversity. For example, every symbol is transmitted $M$ times. As it is mentioned earlier that intervals between the transmitted symbols should be at least coherence time to ensure that different copies of the same symbol undergo independent fading. Maximum ratio combiner can be used to obtain optimal combiner (LARSSON,2014). If we send the same symbol M times then it applies the $(\mathrm{M}, \mathrm{I})$ repetition code. We can also use non-trivial coding. Error control coding and interleaving is an effective way to combat time selective or fast fading.

\section{System Simulation and Data Analysis}

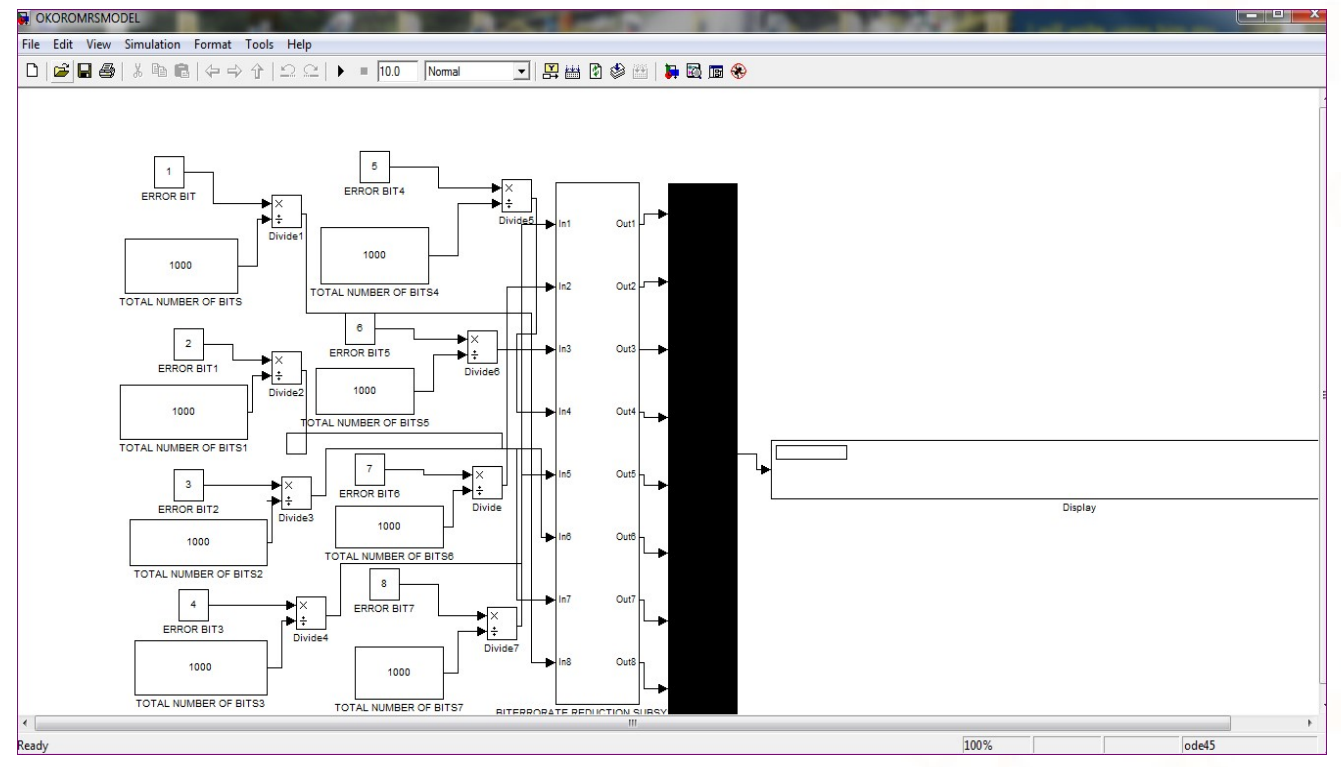

FIG 1 Designed model for bit error rate reduction

Fig 1 shows the designed model for bit errorrate reduction which helps to stop interference in communication industry thereby enhancing free network. 


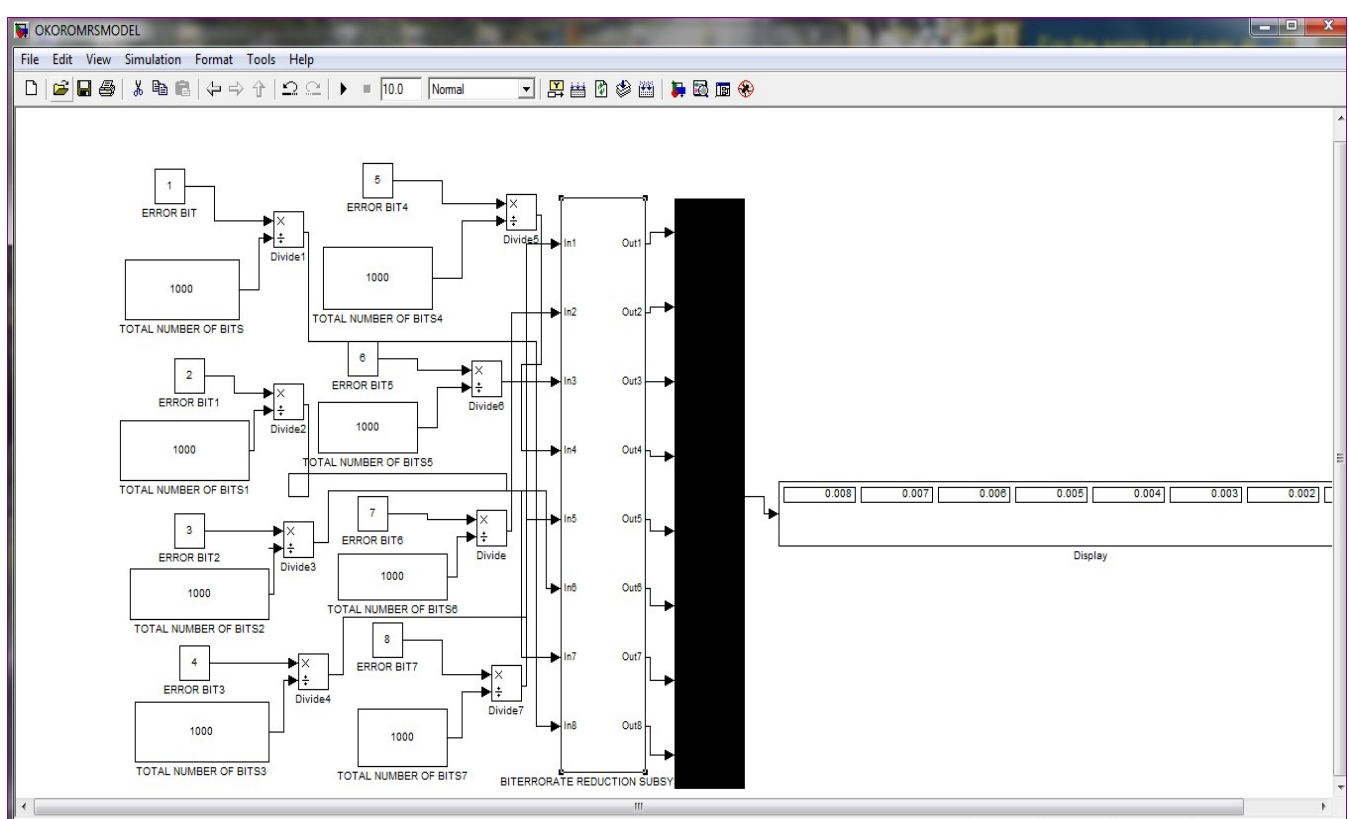

FIG 2 Implemented Model for Bit Error Rate Reduction in enhancing the channel capacity of a celluler mobile system using frequency diversit

Fig 2 shows the bit errror rate reduction model which enhances the efficiency of channel capacity of a celluler mobile system using frequency diversity. This stops call drop,interference, fading to mention a few.

\section{Result Analysis}

TABLE 1 CAPACITY VS FREQUENCY

\begin{tabular}{|c|c|}
\hline CAPACITY & FREQUENCY \\
\hline 4.76 & 100 \\
\hline 47.6 & 200 \\
\hline 476 & 300 \\
\hline 4760 & 400 \\
\hline 47600 & 500 \\
\hline 476000 & 600 \\
\hline
\end{tabular}

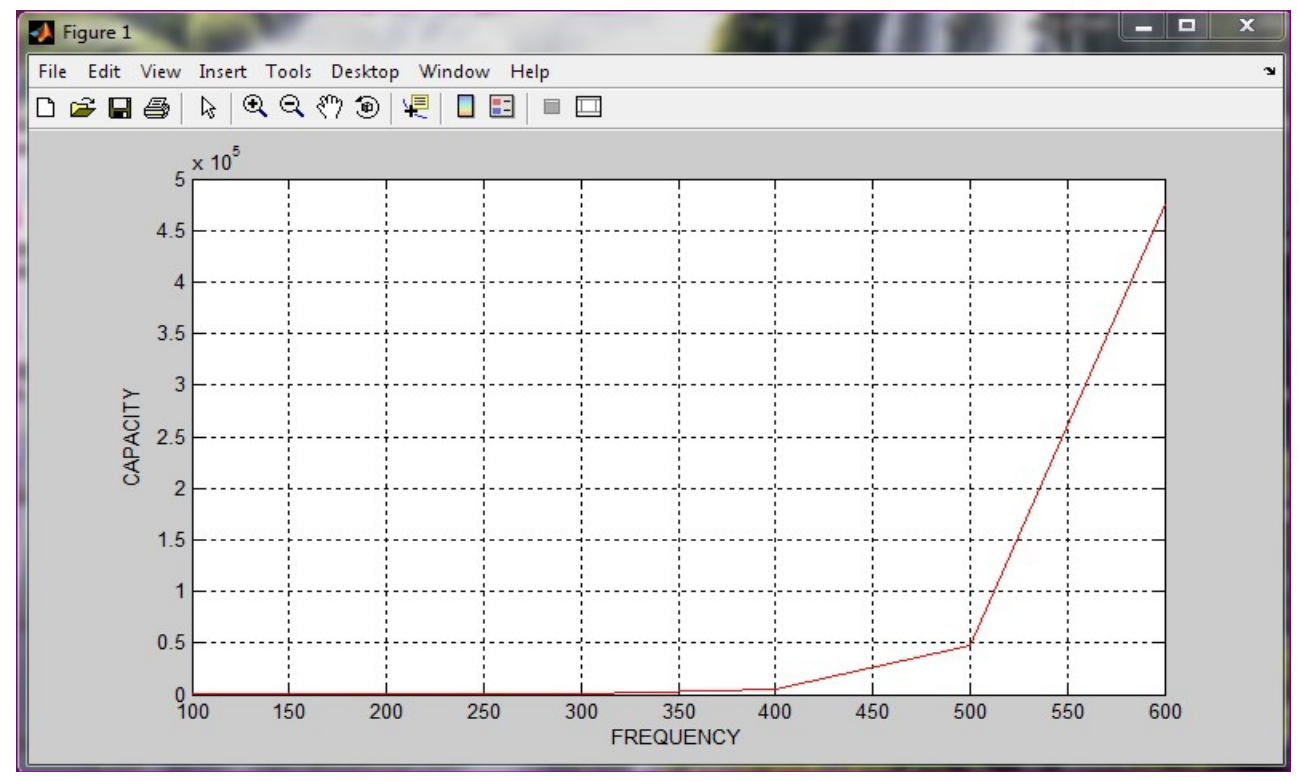

FIG 3: CAPACITY VS FREQUENCY 
International Journal of Trend in Scientific Research and Development (IJTSRD) ISSN: 2456-6470

Fig 3 Shows capacity vs frequency graph.This shows that as the capacity increases the frquency diversity equally increases hence forth minimizing interference,fading to mention a few thereby increasing free network.

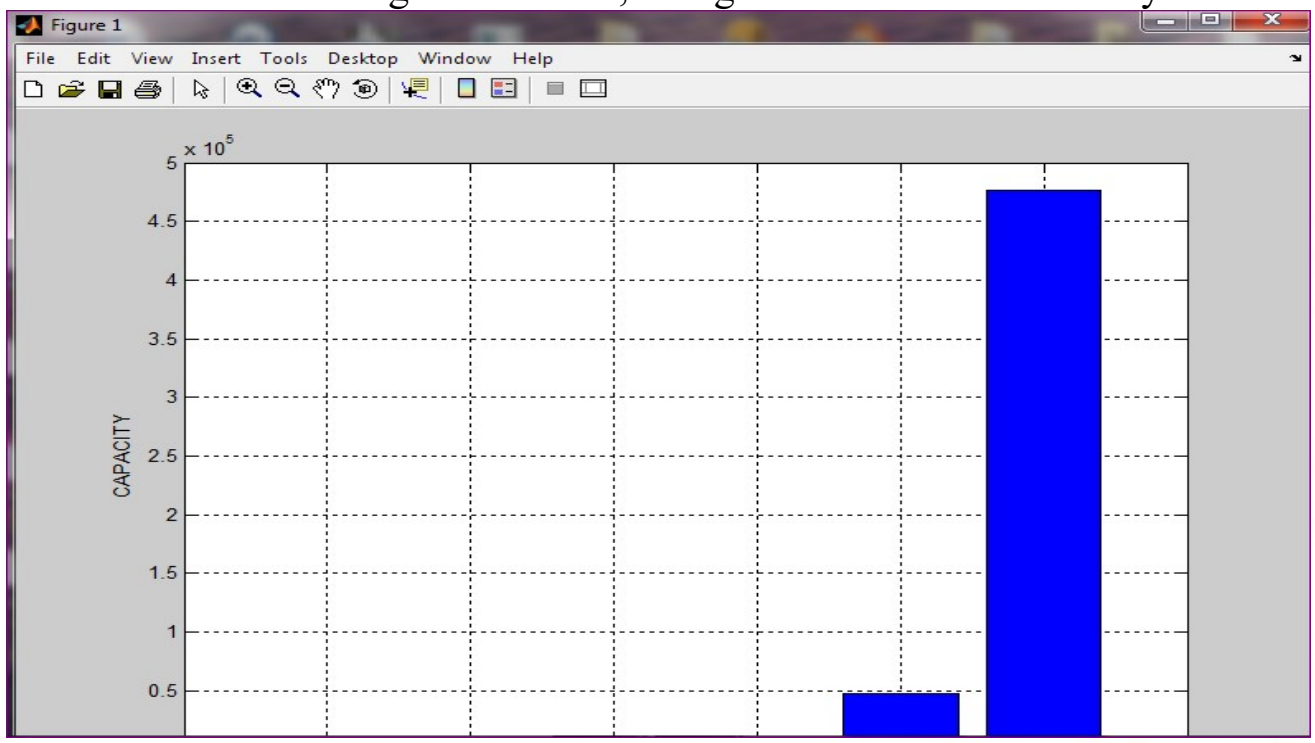

FIG 4: BAR CHART OF CAPACITY VS FREQUENCY

Fig 4 show bar chart of capacity vs frequency. This shows that as the capacity increases the frequency equally increases showing perfect free network for the subscribers without an atom of interference and fading.

Table 2: BIT ERROR RATE VSTIME

\begin{tabular}{|c|c|}
\hline Bit error rate & Time (s) \\
\hline 0.008 & 1 \\
\hline 0.007 & 2 \\
\hline 0.006 & 3 \\
\hline 0.005 & 4 \\
\hline 0.004 & 5 \\
\hline 0.003 & 6 \\
\hline 0.002 & 7 \\
\hline 0.001 & 8 \\
\hline
\end{tabular}

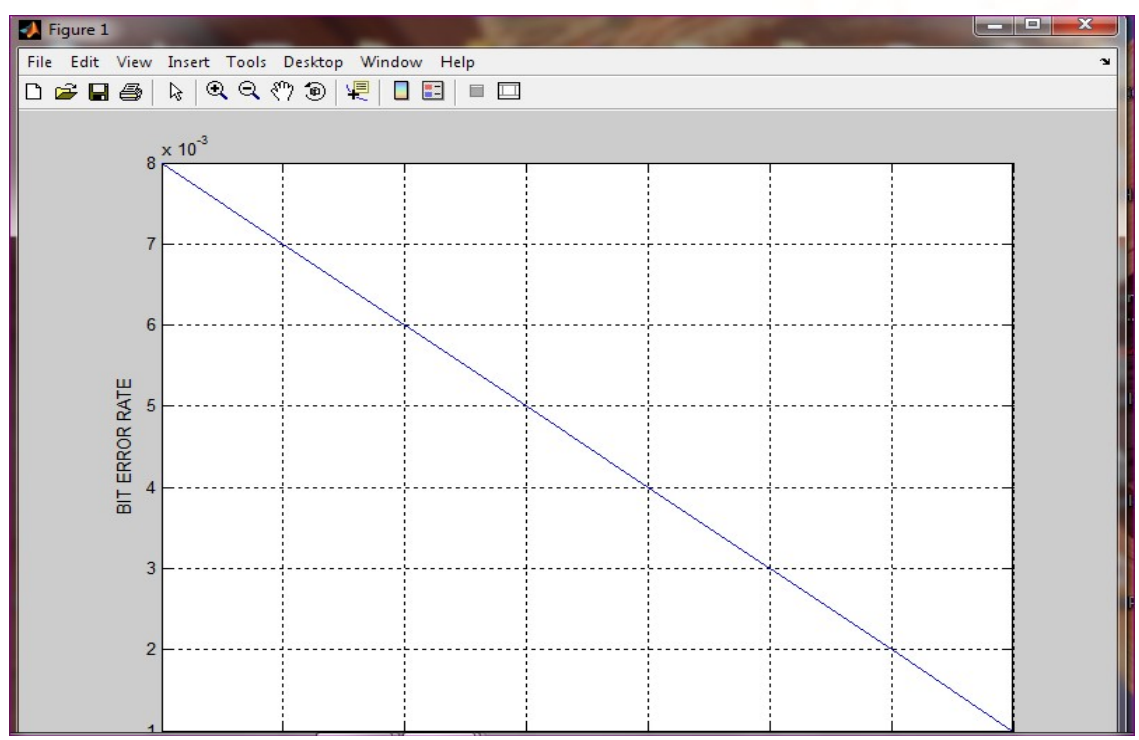

FIG 5: BIT ERROR RATE VS TIME 
Fig 5 Shows the graph of bit error rate vs time.This shows that the bit error rate is inversely proportional to the time. The result shows that the bit error rate decreases as the Time increases thereby enhancing the efficiency of free network in enhancing channel capacity of a celluler mobile system using frequency diversity.

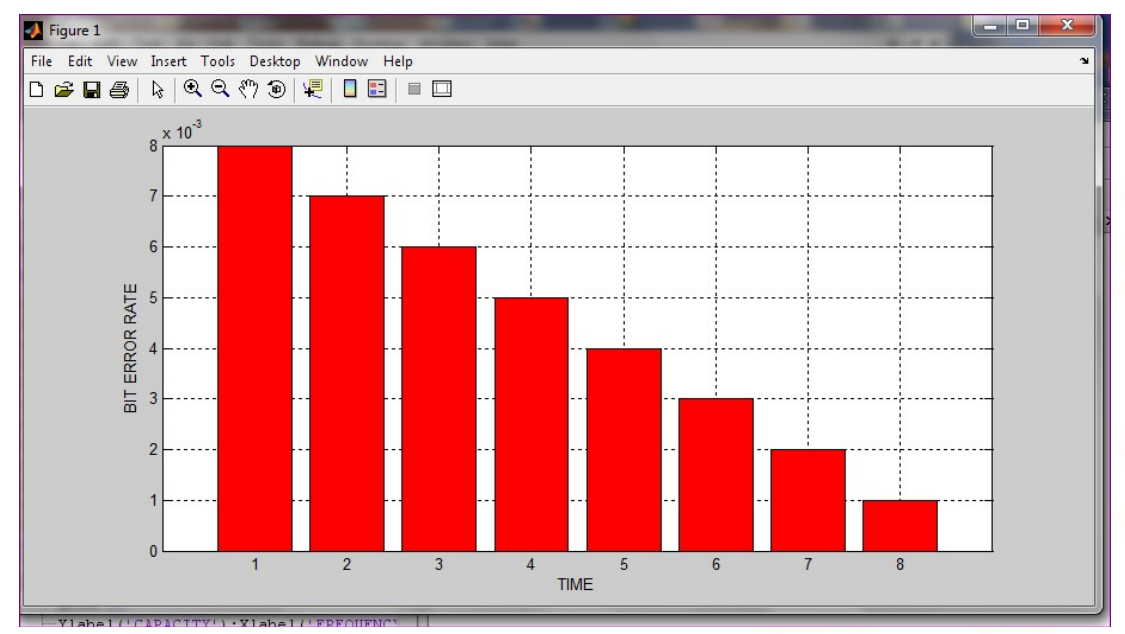

\section{FIG 6: BAR CHART FOR BIT ERROR RATE VS TIME}

Fig 6 shows the bar chart for bit error rate vs time. The chart shows that the bit error rate decreases as the time increases resulting to efficiency of quality of the network.

\subsection{Conclusion}

Communication is an epitome of passage of piece of information from source to sink and to maintain it without interference, fading, call drop thereby enhancing channel capacity of a celluler mobile system using frequency diversity; is by evaluating the channel capacity at frequency diversity, evaluating bit error rate in channel capacity at frequency diversity, ensuring multipath or frequency diversity, ensuring spatial/space diversity, transmitted and received diversity, determine time/temporal diversity and designing a model that reduces bit error rate after simulation in matlab.

\section{REFERENCES}

1. T.M. Duman, -Time A. Ghrayeb, Block "Space Codes," in Co Communication Systems, John Wiley and Sons, 2015, PP.71-72

2. T.M. Duman, A. Ghrayeb, "Need for MIMO Communication Systems, John Wiley and Sons, 2015, PP.1-2

3. Z. Liu, G.B. Giannakis, - Time Coding B for. Broadband Muquet, Wireless S. Zho Communication," USA, 2014.

4. M. S. Ullah, M. J. Uddin, "Performance Using Alamout is Scheme and Maximum Ratio Combining 2016.
5. P.M. Chidambara Nathan, "History of Communications, PHI Press, PP.1-29( 2014)

6. "History and Evolution cations of the mob Businessman Globalization of mobile and Wireless technology, Springer, PP.48-49.

7. Sarkar, Tapan K. Salazar-Palma, Magdalena Multiple Mokole,-Input-Multiple -Eric L Output (MIMO) Antenna Systems," in Physics of Multi anten Processing, Hoboken, NJ, USA ,2014, pp 172-173.

8. Tech Target, (November, 2014). SIMO single input multiple output [online], Available: http://searchmobilecomputing. techtarget.com/definition/SIMO.

9. Y. -W. Peter Hong, Wan-Jen Huang, C. -C. Jay Kuo, "review of wi and MIMO technique," in Cooperative Communication. 26-35.(2015)

10. Erik G larsson, petrestoica, "MIMO informationTime Theory," Block Coding in for Wireless Communication, Cambridge UK, Cambridge University Press, 2014. pp 4-7

11. AvClaudeOestges, Bruno Clerckx, "Brie wireless communications: from real-world propagation to space-time code design, Academic press,2015, pp $1-3$

12. Ilan Hen, "MIMO Architecture for Wireless Volume 10 Issue 02 , May 15, 2016. 
International Journal of Trend in Scientific Research and Development (IJTSRD) ISSN: 2456-6470

13. A. Neubauer, J. Freudenberger," in Coding V. Kunn, the Algorithms, Architectures and Applications, John Wiley and Sons, 2014, PP.17

14. Berta Delango, "Performance-Time Block Coding Evaluation MIMO Communication System," School Mathematics, of Computer Linnaeus University, Vaxjo, Feb. 2013

15. Sarkar, Tapan K. Salazar-Palma, Magdalena Mokole, Eric L Maxwellian Viewpoint,"PhysicsofMultiantennaSystemsin and Broadband Processing, Hoboken, NJ, USA: Wiley, 2014. pp 114-116

16. Thomas M. Cover, Joy A. Thomas, "En Information," in Elements of Information The pp12-23. (2015)

17. Erik G larsson, petrestoica,ceTimeBlockCoding"MIMO in for Wireless Communication, Cambridge UK, Cambridge University Press, 2015. pp 22-25.

18. Jankiraman, - Time Mohinder, Block Space Coding, "Space-Time Codes and MIMOin Systems, Norwood, MA, USA, Artech House , July 2014. pp 103-132.

19. On MIMO systems and Adaptive array for wireless communication, MattiasWennstrom, Uppsala University, 2015 .pp 35 - 36.

20. Ghrayeb, Ali Duman, TolgaCapacityandM,Information" Rates of MIMO Channels," in Coding for MIMO Communication Systems. Hoboken, NJ, USA, Wiley, 2014 pp 4550.

21. A. Neubauer, J. Freudenberger, V. Kunn, "Information The Algorithms, Architectures and Applications, John Wiley and Sons, 2014, PP.17

22. David Gesbert, MansoorShafi, Da-shanShiu, Peter J. Smith, Theory to Practice: An Overview of MIMO Space-Time Coded Wireless Sys Areas In Communications, Vol. 21, NO. 3, APRIL 2013.

23. R. L. Freeman, "Radio propagation And Fa. Telecommunications, John Wiley and Sons, 2015, PP.19-22

24. T.M. Duman, A. Ghrayeb, "Fading Channels and Dive MIMO Communication Systems, John Wiley and Sons, 2015, PP.20-22
25. C.B. Dietrich, K. Dietze, J. R. Nealy, W. L. Stutzman, "Spatial, polarizat Diversity for Wireless Handleld Terminals" 2014

26. Vikrant Vij, "Diversity" in Wireless C Delhi, 2010, PP.24

27. D. Popovic, Z. Popovic,Multibeam " Antennas with Polarization and Angle Vol NO, 8, 2014

28. M. Kar,-Branch P. Wahid, Space "Two and Polarization Di

29. A. G. Dabak, S. Hosur, Acomparison T. Schmidl, of the open loop transmit C. Sengup diversity schemes for third generation wireless Systems," 2015

30. M. Jankiraman, "MIMO- Time Wireless Trellis Codes and Channel" MIMO Systems, Artech House, 2014 